\title{
Macroscopic Modeling of Magnetic Microwires for Finite Element Simulations of Inductive Components
}

\author{
Alberto Delgado, Student Member, IEEE, Jesús Ángel Oliver, Member; IEEE, \\ José Antonio Cobos, Fellore IEEE, and Jorge Rodriguez-Moreno
}

\begin{abstract}
New compound magnetic material based on parallel continuous ferromagnetic microwires (e.g., based on cobalt) embedded in a body made of polymeric material can potentially offer significant mechanical advantages over well-known fragile ferrite in some applications where the magnetic material has to withstand mechanical vibrations. The diameter of the magnetic microwires is minimal $(3-60 \mu \mathrm{m})$ compared to the rest of the dimensions $(80-300 \mathrm{~mm})$ and the number of microwires is in the order of $1000 \mathrm{~s}$. It requires extremely fine meshes, then brute force simulation by the finite element method is not possible. In this article, a method to overcome this limitation is proposed, which is based on the substitution of the original material by a homogenous material that behaves, in a macroscopic sense, as the compound material. The physical properties of the homogeneous material are obtained in different spatial directions, due to the inherent anisotropy of the compounded magnetic component, through analytical equations.
\end{abstract}

Index Terms-Anisotropic model, antennas, compounds magnetic material, finite element (FE) analysis, homogeneous model, magnetic components, microwires, power electronics, radio frequency identification.

\section{INTRODUCTION}

$\mathbf{T}$ $\mathrm{HE}$ fragility of ferrite as core material for inductive com ponents poses significant challenges from the mechanical point of view. Therefore, a significant effort has been made to protect the magnetic components: housing potting, and overmoulding. This means an increase in manufacturing and design time, and costs.

The proposal of new compounded magnetic materials using magnetic microwires can offer significant mechanical advantages over conventional ferrites whilst maintaining good magnetic properties [see Fig. 1(a)]. This advantage can be exploited to simplify, improve the reliability, and reduce the cost of those applications in which the magnetic components are exposed to mechanical vibrations or impacts [see Fig. 1(b)]. There-fore, numerous investigations on flexible magnetic materials are emerging, such as the microwires [1] [5].

Due to the inherent anisotropic nature of the compound mag-netic material [see Fig. 4(a)], direct analytical equations from the literature [6] [10) for calculating the inductance and losses are not feasible. (a)

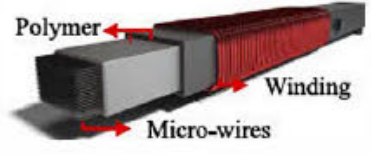

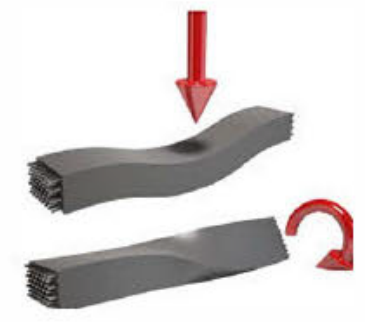

(b)
Fig. 1. Illustration of a prototype designed by Premo Group of an RFID based on microwires.

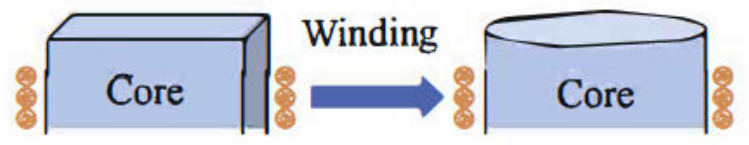

Fig. 2. Illustration of the transformation to 3-D to 2-D configuration done in [11] and [12].

Although brute force analysis by three dimensional (3 D) finite element (FE) simulations requires high computational cost, especially when the amount of microwires is elevated, and its diameter is minimal because the mesh required to perform the simulation is excessively fine. Furthermore, the 3-D model can be expressed as the 2-D model [11], [12] (see Fig. 2) and 2-D FE simulations in the $X Y$ plane, which are based on cartesian coordinates, would be possible. Fig. 3(a) shows the FE configuration. In this case, the input current of the antenna must be defined in the azimuthal direction. This simulation allows us to obtain the energy of the antenna and the losses of the microwires, but we cannot obtain the losses of the copper winding. On the other hand, 2-D FE simulation in the $R Z$ plane, which is based on cylindrical coordinates, would be possible if it were a single microwire, as can be seen in Fig. 3(b). This finite element configuration provides the energy and the losses of the single microwire and the copper winding losses.

Regarding the FE simulations in $R Z$ plane, this article presents a method to allow FE analysis of inductive components made of microwires as a magnetic core. The proposed method is based 


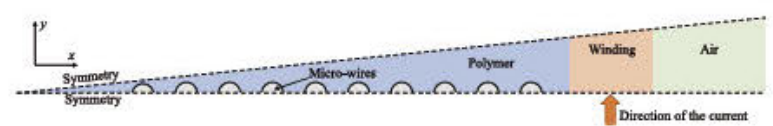

(a)

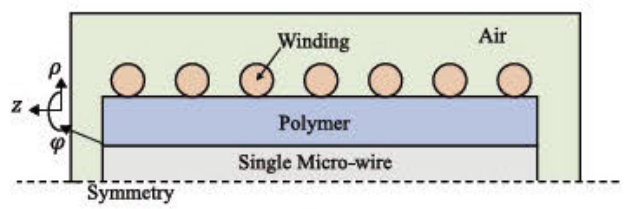

(b)

Fig. 3. Illustration of different 2-D FE configuration that allows the simulation of the microwires.

on the homogenization of the microwires. The homogenization process starts with the replacement of the magnetic microwires, with their magnetic and electrical properties, and the surrounding media (that has unit permeability) by an equivalent material, which will have new magnetic and electrical properties. Based on previous work [13], we simplify the homogenization process; instead of obtaining the magnetic and electrical properties in several steps, we propose only one equation for each parameter.

Thus, due to the anisotropic behavior of the composite magnetic material, a matrix describing the modification of the physical properties in all the relevant directions is needed. The proposed model provides a complex permeability in the longitudinal direction: the real part will provide the energy and the imaginary part the losses; a complex permeability in the radial direction that will conserve the energy and the losses; and a conductivity in the angular direction that will give rise to the losses due to the eddy currents.

This article is structured into four sections. In Section II, the modeling of the microwires is described. First, we describe the physical properties of the polymer and the magnetic microwires, and then, we develop the modeling into two parts. We homogenize the microwires when 1) they are excited in their longitudinal direction, and 2) they are excited in their perpendicular direction. In Section III, we obtain the magnetic and electrical properties of the microwires. In Section IV, we compare the measurements and the FE results using our proposed model.

\section{Fundamental Analysis of the Physical Properties}

A model describing the equivalent physical properties of the magnetic microwires in all directions [see Fig. 4(a)] will be developed to allow the simulation of these materials by FE tools. The model will be developed using the following assumptions.

1) Axial symmetry, as shown in Fig. 4(b), the magnetic field lines in a cylindrical solenoid (or antenna) will have components both in the axial $z$ - and radial $\rho$-direction and the current density field will have only angular component $\varphi$.

2) Polymeric material has the following characteristics:

a) relative permeability: $\mu_{p}=1$;

b) conductivity: $\sigma_{p}=0 \mathrm{~S} / \mathrm{m}$;

c) equivalent radius: $r_{p}=\sqrt{r_{h}^{2}-N_{\mu w} r_{\mu w}^{2}}$.

3) Microwires have the following characteristics.

a) Complex relative permeability: $\mu_{\mu w}=\mu_{\mu w}^{\prime}-j \mu_{\mu w}^{\prime \prime}$, where $\mu_{\mu w}^{\prime}$ is the real part of the permeability, and it is related to the energy; and $\mu_{\mu w}^{\prime \prime}$ is the imaginary part

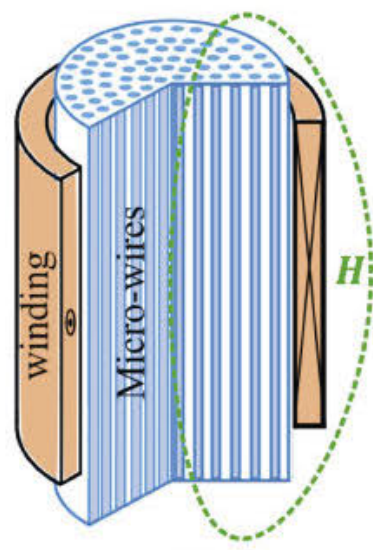

(a)

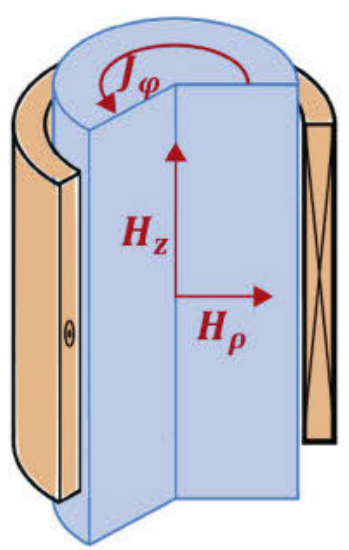

(b)
Fig. 4. (a) 3-D view of a magnetic core made of microwires immersed in magnetic field $H$. (b) 3-D view of a homogeneous magnetic core immersed in magnetic decomposed field $H$.

of the permeability, and it is related to the hysteresis losses.

b) Conductivity: $\sigma_{\mu w}$.

c) Radius: $r_{\mu w}$.

4) The homogeneous material will keep the same geometrical dimensions as the actual compound and will have the following characteristics:

a) complex permeability in $z$-direction: $\mu_{h z}=\mu_{h z}^{\prime}-$ $j \mu_{h z}^{\prime \prime}$, where the real component of the permeability is related to the energy; and the imaginary part describes the hysteresis losses;

b) complex permeability in $\rho$-direction: $\mu_{h \rho}=\mu_{h \rho}^{\prime}-$ $j \mu_{h \rho}^{\prime \prime}$

c) conductivity in $\varphi$-direction: $\sigma_{h \varphi}$;

d) radius $r_{h}$ that is equal to the total radius of the core.

\section{A. Modeling of Microwires With Magnetic Field Excitation in Longitudinal Direction}

Assuming uniform longitudinal magnetic field $H_{z}$ that excites the magnetic core, the magnetic, and electrical properties of the homogeneous material that are involved in this direction (the complex permeability $z$ and conductivity $\varphi$ ) are calculated in such a way that the losses and the magnetic energy will be kept the same as in the homogeneous material.

The concerned magnetic properties are: complex permeability in the longitudinal direction that is called $\mu_{h z}=\mu_{h z}^{\prime}-j \mu_{h z}^{\prime \prime}$ to keep the same energy and hysteresis losses. Also, due to the induced currents created by the magnetic field in this direction, a conductivity $\sigma_{h \varphi}$ has to be defined to keep the same eddy current losses.

Assuming that the behavior of the microwires composite is linear and anisotropic (depends on the direction), it is possible to apply superposition and to analyze the problem analyzing the impact of the magnetic in the $z$ - and $\rho$-direction independently. Therefore, for the magnetic field $H_{z}$, it is possible to use the analytical solution for the impedance of an infinite cylindrical magnetic core, which is stimulated by a uniform surface current density [9], [13]. Since this equation is valid only under the assumption of the infinitely long core and the leakage field has no contribution in this direction, and therefore, it is not relevant only for this particular case. However, if we compared the length of 
a Radio Frequency IDentification antenna (RFID) antenna that goes from 80 to $300 \mathrm{~mm}$, with the total radius of the microwires composite (around $1.2 \mathrm{~mm}$ ), we could assume that the magnetic core is infinitely long.

The equivalent impedance of a magnetic core of length $l$ and radius $r$, relative permeability $\mu_{r}$, and skin depth $\delta$ with 1-turn can be calculated as

$$
Z=\frac{j \omega \mu_{r} \mu_{0} r^{2} \pi}{l} F(\Delta)
$$

where

$$
F(\Delta)=\frac{2 I_{1}(\sqrt{2 j} \Delta)}{\sqrt{2 j} \Delta I_{0}(\sqrt{2 j}) \Delta}
$$

where $I_{n}$ is the modified Bessel functions of the $n$ kind and $\Delta=r / \delta$.

Since these kinds of components work from 20 to $120 \mathrm{kHz}$ and the diameter of the magnetic microwires is around $10 \mathrm{~s}$ of $\mu \mathrm{m}$, it is possible to assume $\Delta \leq 1$. Then, (2) can be simplified to

$$
F(\Delta)=1-j \frac{\Delta^{2}}{4}=F_{R}(\Delta)-j F_{I}(\Delta) .
$$

The self-impedance of the $N_{\mu w}$ magnetic microwires of radius $r_{\mu w}$ is given by (1)

$$
\begin{aligned}
Z_{\mu w}= & N_{\mu w} \frac{j \omega\left(\mu_{\mu w}^{\prime}-j \mu_{\mu w}^{\prime \prime}\right) \mu_{0} r_{\mu w}^{2} \pi}{l} \\
& \times\left(F_{R}(\Delta \mu w)-j F_{I}(\Delta \mu w)\right)
\end{aligned}
$$

where (4) can be decomposed to real and imaginary parts

$$
\begin{aligned}
Z_{\mu w}^{I} & =N_{\mu w} \frac{j \omega\left(\mu_{\mu w}^{\prime} F_{R}(\Delta \mu w)-\mu_{\mu w}^{\prime \prime} F_{I}(\Delta \mu w)\right) \mu_{0} r_{\mu w}^{2} \pi}{l} \\
Z_{\mu w}^{R} & =N_{\mu w} \frac{\omega\left(\mu_{\mu w}^{\prime} F_{I}(\Delta \mu w)+\mu_{\mu w}^{\prime \prime} F_{R}(\Delta \mu w)\right) \mu_{0} r_{\mu w}^{2} \pi}{l}
\end{aligned}
$$

where the imaginary component (5a) represents the energy, and the real component $(5 b)$ represents the losses.

The self-impedance of the polymeric material part that occupies an area $A_{p}=r_{p}^{2} \pi$ is given by (1) and it has only one component (assuming that the conductivity of the polymer is zero)

$$
Z_{p}=\frac{j \omega \mu_{p} \mu_{0} r_{p}^{2} \pi}{l} F_{R}\left(\Delta_{p}\right) .
$$

The self-impedance of the homogeneous compound with radius $r_{h}$ [see Fig. 4(b)] is given by (1)

$$
Z_{h}=\frac{j \omega\left(\mu_{h}^{\prime}-j \mu_{h}^{\prime \prime}\right) \mu_{0} r_{h}^{2} \pi}{l}\left(F_{R}\left(\Delta_{h}\right)-j F_{I}\left(\Delta_{h}\right)\right)
$$

where (7) can be decomposed in real and imaginary parts

$$
\begin{aligned}
Z_{h}^{I} & =\frac{j \omega\left(\mu_{h}^{\prime} F_{R}\left(\Delta_{h}\right)-\mu_{h}^{\prime \prime} F_{I}\left(\Delta_{h}\right)\right) \mu_{0} r_{h}^{2} \pi}{l} \\
Z_{h}^{R} & =\frac{\omega\left(\mu_{h}^{\prime} F_{I}\left(\Delta_{h}\right)+\mu_{h}^{\prime \prime} F_{R}\left(\Delta_{h}\right)\right) \mu_{0} r_{h}^{2} \pi}{l}
\end{aligned}
$$

where the imaginary component (8a) represents the energy, and the real component $(8 \mathrm{~b})$ represents the losses.

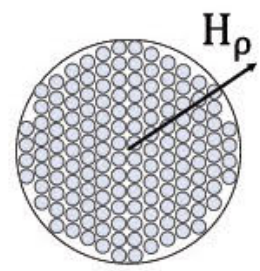

(a)

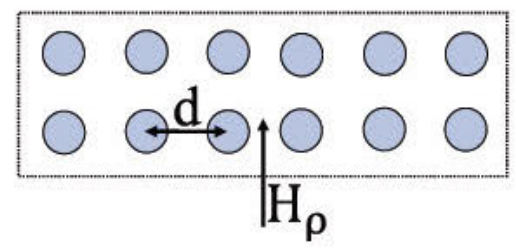

(b)
Fig. 5. Illustration of: (a) cross-section of compound material; and (b) detailed cross-section.

Since the magnetic energy of the magnetic microwires and the polymer must be equal to the magnetic energy of the compound, the imaginary part of its impedances [see (5a), (6), and (8a)] are equalized

$$
\begin{aligned}
& N_{\mu w}\left(\mu_{\mu w}^{\prime} F_{R}(\Delta \mu w)-\mu_{\mu w}^{\prime \prime} F_{I}(\Delta \mu w)\right) r_{\mu w}^{2}+r_{p}^{2} F_{R}\left(\Delta_{p}\right) \\
& \quad=\left(\mu_{h}^{\prime} F_{R}\left(\Delta_{h}\right)-\mu_{h}^{\prime \prime} F_{I}\left(\Delta_{h}\right)\right) r_{h}^{2} .
\end{aligned}
$$

Assuming imaginary part of the permeability smaller than the real part, that is, $\mu_{\mu w}^{\prime} \geqslant \mu_{\mu w}^{\prime \prime} F_{I}\left(\Delta_{\mu w}\right)$, from (9), the real permeability of the homogeneous material can be expressed as

$$
\mu_{h z}^{\prime}=N_{\mu w} \mu_{\mu w}^{\prime}\left(\frac{r_{\mu w}}{r_{h}}\right)^{2}+\left(1-N_{\mu w}\left(\frac{r_{\mu w}}{r_{h}}\right)^{2}\right) .
$$

Since the losses of the magnetic microwires must be equal to the losses of the compound, the imaginary part of its impedances [see $(5 b)$ and $(8 b)]$ are equalized

$$
\begin{aligned}
& N_{\mu w}\left(\mu_{\mu w}^{\prime} F_{I}(\Delta \mu w)+\mu_{\mu w}^{\prime \prime} F_{R}(\Delta \mu w)\right) r_{\mu w}^{2} \\
& =\left(\mu_{h}^{\prime} F_{I}\left(\Delta_{h}\right)+\mu_{h}^{\prime \prime} F_{R}\left(\Delta_{h}\right)\right) r_{h}^{2} .
\end{aligned}
$$

Since the term that is multiplied by $F_{R}$ depends only on the imaginary permeability, it can be noticed that represents the hysteresis losses. The term multiplied by $F_{I}$ depends on the skin effect, therefore, represents the losses per eddy current. Then, to keep the same losses due to eddy current and hysteresis, (11) can be divided into two equations

$$
\begin{gathered}
N_{\mu w}\left(\mu_{\mu w}^{\prime \prime} F_{R}(\Delta \mu w)\right) r_{\mu w}^{2}=\left(\mu_{h}^{\prime \prime} F_{R}\left(\Delta_{h}\right)\right) r_{h}^{2} \\
N_{\mu w}\left(\mu_{\mu w}^{\prime} F_{I}(\Delta \mu w)\right) r_{\mu w}^{2}=\left(\mu_{h}^{\prime} F_{I}\left(\Delta_{h}\right)\right) r_{h}^{2} .
\end{gathered}
$$

Regarding (12a), the imaginary part of the relative permeability of the homogeneous compound can be expressed as

$$
\mu_{h z}^{\prime \prime}=\mu_{\mu w}^{\prime \prime}\left(\frac{r_{\mu w}}{r_{h}}\right)^{2} N_{\mu w} .
$$

From (12b), the conductivity of the homogeneous material can be calculated as

$$
\sigma_{h \varphi}=\sigma_{\mu w}\left(\frac{\mu_{\mu w}^{\prime}}{\mu_{h}^{\prime}}\right)^{2}\left(\frac{r_{\mu w}}{r_{h}}\right)^{4} N_{\mu w} .
$$

\section{B. Modeling of Microwires With Magnetic Field Excitation in Perpendicular Direction}

In the case that the magnetic flux density is in the radial direction [see Fig. 5(a)], it will find microwires of high permeability separated by a polymer of relative permeability equal to one. 


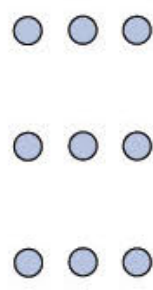

(a)

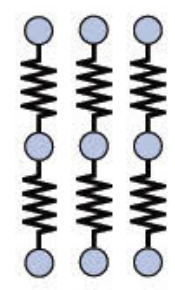

(b)

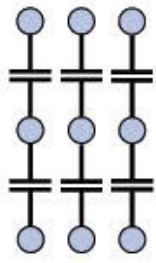

(c)
Fig. 6. Schematic of: (a) array of microwires in an elementary cell of polymer; (b) magnetic reluctance model circuit $\left(R_{i}\right)$; (c) dielectric capacitive model circuit $\left(C_{i}\right)$.

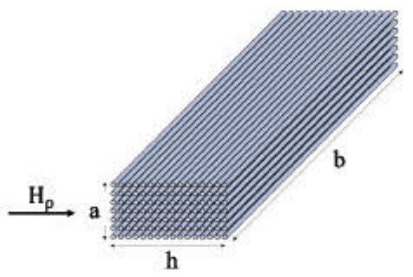

Fig. 7. Illustration of a rectangular magnetic core made of macroscopic microwires excited by a 1-D magnetic field.

Therefore, the resultant permeability in this direction will be very low.

If we take a closer look [see Fig. 5(b)], the radial field problem can be considered as an $X Y$ field problem. In this article, an expression for real relative permeability is described based on the capacitance analogy [14], [15] using the results of a set of electrostatic FE simulations with Ansys Maxwell.

1) Real Part of the Complex Permeability: Since the permeability of the microwires is 1000 s of times higher than the permeability of the polymer [see Fig. 6(a)], it will be assumed that the reluctance $R_{i}$ [see Fig. 6(b)] of this path can be calculated as the capacitance $C_{i}$ [see Fig. 6(c)] between the microwires analogue, as the calculation of the gaps in inductor or transformer

$$
\begin{aligned}
C_{i} & =\epsilon_{0} F(g) \\
R_{i} & =\frac{1}{\mu_{0} F(g)}
\end{aligned}
$$

where $\mu_{0}$ is the vacuum permeability, $\epsilon_{0}$ is the vacuum permittivity, and $F(g)$ is a geometry function.

Assuming the microwires have an infinite relative permeability, to estimate the variation of the capacitance with the radius of the microwires $r_{\mu w}$ and their distance $d$ [see Fig. 5(b)], we performed a set of electrostatic 2-D simulations in Ansys Maxwell by sweeping $r_{\mu w}$ and $d$ to calculate the capacitance. Based on the results of these different tests, the empirical equation (16) to calculate the capacitance is obtained as a function of the dimensionless parameter $r_{\mu w} / d$

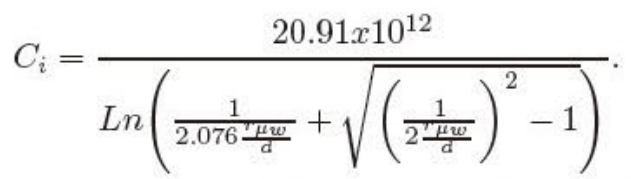

Under the assumption of a homogeneous flux density distribution in the array of microwires, as shown in Fig. 7, the total homogeneous reluctance can be calculated as

$$
R_{h}=\frac{h}{\mu_{0} \mu_{h \rho}^{\prime} b a}
$$

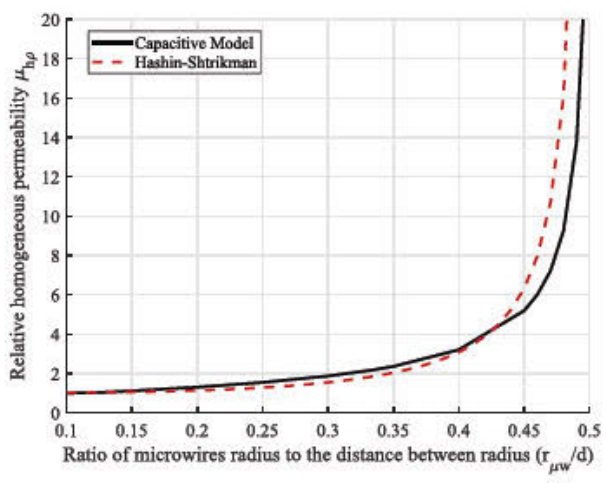

Fig. 8. Relative homogeneous permeability as a function of the $r_{\mu w} / d$ calculated with the capacitance model compared with the Hashin-Shtrikman model [16].

where $\mu_{\rho h}^{\prime}$ is the real relative permeability of the homogeneous microwire. To obtain this $R_{h}$, we must calculate the equivalent reluctance of the magnetic circuit [see Fig. 6(b)]. If the reluctance of an air gap between two microwires, with the assumption that the microwires have an infinity relative permeability, can be expressed as

$$
R_{i}=\frac{\epsilon_{0}}{\mu_{0} C_{i} b} .
$$

We can define the reluctance between rows as

$$
R_{i r}=R_{i} \frac{d}{a}
$$

where $d$ is the distance between microwires [see Fig. 5(b)].

Then, it is possible to define the reluctance total as

$$
R_{h}=R_{i r}(n-1)=R_{i r}\left(\frac{h}{d}-1\right)=R_{i} \frac{h-d}{a} .
$$

Accordingly, under the assumption that the distance $h$ is larger than the distance $d$ and substituting (18) in (20), we can reduce (20) to

$$
R_{h}=\frac{\epsilon_{0} h}{\mu_{0} C_{i} b a} .
$$

If (17) and (21) are equated, we can obtain the real homogeneous permeability as a function of the dimensionless parameter $r_{\mu w} / d$, as shown in (22), and Fig. 8 shows the results compared with the well-known equation of Hashin-Shtrikman [16], which describes the theoretical determination of the magnetic properties of macroscopically homogeneous multiphase materials that depends on the volume fractions and the permeabilities of the materials involved where the magnetic material has a random distribution

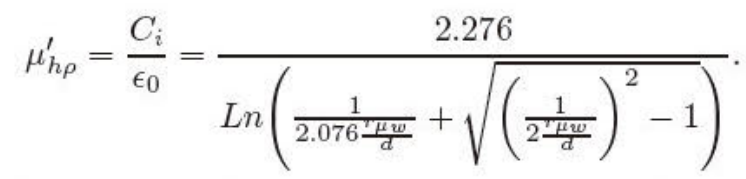

2) Imaginary Part of the Complex Permeability: The real and imaginary parts of the permeability are related by the Kramers-Kronig relation. Due to the linear, time-invariant, and casual relation between magnetization and magnetic field, the imaginary parts of relative permeability can be calculated from the real relative permeability [17]. 


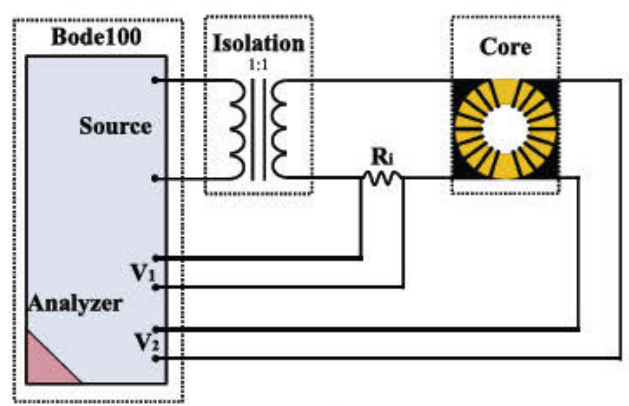

(a)

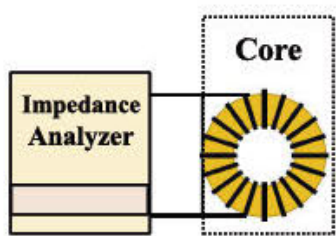

(b)

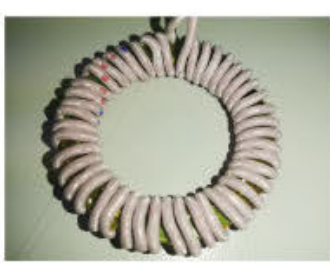

(c)
Fig. 9. Illustration. (a) schematic of the characterization setup with Bode100; (b) schematic of the characterization setup with the Impedance Analyzer; (c) toroid used to characterize the microwires.

Therefore, the homogeneous imaginary permeability in the radial direction using the Hilbert transform can be expressed as

$$
\mu_{h \rho}^{\prime \prime}=-\frac{2}{\pi} \quad{ }_{0}^{\infty} \frac{\mu_{h \rho}^{\prime}(x)}{x-\omega} d x
$$

where $\omega$ is the angular frequency.

\section{MICROWIRES CHARACTERIZATION AND PARAMETER ESTIMATION}

To validate the proposed models, we used an alloy of amorphous magnetic microwire based on cobalt ( $\mathrm{Co}-\mathrm{Fe}-\mathrm{B}-\mathrm{Si}-\mathrm{Ni}-$ $\mathrm{Mn}$ ) from Wire Machine Technologies. The manufacturers do not have information about the magnetic properties, then the magnetic properties of this material have been extracted and compared using small-signal analysis. The method has been performed in two diverse ways to validate the measurements: using a Vector Network Analyzer (Bode100 from Omicron) [see Fig. 9(a)] and using an impedance analyzer [see Fig. 9(b)].

These microwires have isotropic behavior. These kinds of microwires are isolated, the diameter of the microwire is around $33.7 \mu \mathrm{m}$ and with the coating is approximately $35.5 \mu \mathrm{m}$.

\section{A. Vector Network Analyzer}

A toroidal transformer has been built to characterize the microwires. The dimensions of this toroid are as follows: $\mathrm{ID}=40 \mathrm{~mm}, \mathrm{OD}=50 \mathrm{~mm}$, and $h=3.85 \mathrm{~mm}$. In order to minimize the leakage flux, the winding is made with 46-turn of litz-wire conductors. The magnetic core is made of 700 microwires.

The characterization has been performed using the setup shown in Fig. 9(a). The voltage in the secondary and the input current in the primary are measured.

The flux density in a transformer is given by

$$
\frac{d \Phi}{d t}=\frac{v_{2}}{N_{2}}
$$

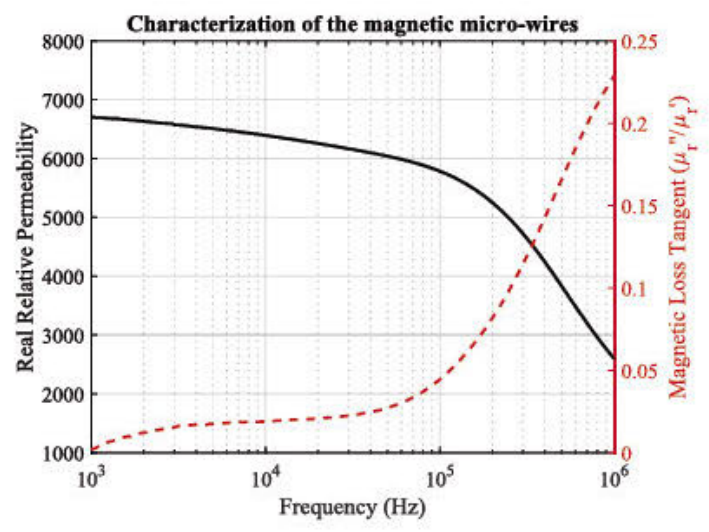

Fig. 10. Measurement of: (a) real relative permeability of the microwires based on cobalt and (b) magnetic loss tangent $\left(\frac{\mu_{\mu w}^{\prime}}{\mu_{\mu w}^{\prime \prime}}\right)$ of the magnetic microwires.

where $N_{2}$ is the secondary turns, and $v_{2}$ is the secondary voltage.

Therefore, the small-signal magnetic field density in the transformer is

$$
j \omega B A=\frac{V_{2}}{N_{2}} .
$$

The magnetic core has two parts: microwires and air/polymer. In this way, (25) can be expressed as

$$
j A_{\mu w} \mu_{\mu w} \mu_{0} H+j A_{\text {air }} \mu_{0} H=\frac{V_{2}}{\omega N_{2}}
$$

where $A_{\mu w}$ and $A_{\text {air }}$ are the total area of the microwires and the total area of air, respectively. Then, $\mu_{\mu w}$ is divided into two components: $\mu_{\mu w}=\mu_{\mu w}^{\prime}-j \mu_{\mu w}^{\prime \prime}$, and $H=N_{1} I_{1} / l_{c}$. The expression (26) can be rewritten as

$$
j\left(\mu_{\mu w}^{\prime}-j \mu_{\mu w}^{\prime \prime}\right)=\frac{V_{2}}{I_{1}}\left(\frac{l_{c}}{\omega N_{1} N_{2} \mu_{0} A_{\mu w}}\right)-j \frac{A_{\text {air }}}{A_{\mu w}}
$$

where $N_{1}$ is the primary turns, $l_{c}$ is the length of the core, and $I_{1}$ is the primary current. Hence, (27) can be divided into two parts: the real part of the relative permeability of the microwires can be given by

$$
\mu_{\mu w}^{\prime}=\operatorname{Im}\left\{\left.\frac{V_{2}}{I_{1}}\right|_{i_{2}=0}\right\}\left(\frac{l_{c}}{\omega N_{1} N_{2} \mu_{0} A_{\mu w}}\right)-\frac{A_{\text {air }}}{A_{\mu w}}
$$

and the imaginary part of the relative permeability of the microwires can be given by

$$
\mu_{\mu w}^{\prime \prime}=\operatorname{Re}\left\{\left.\frac{V_{2}}{I_{1}}\right|_{i_{2}=0}-R_{e}-R_{w}\right\}\left(\frac{l_{c}}{\omega N_{1} N_{2} \mu_{0} A_{\mu w}}\right)
$$

where $R_{w}$ is the ac resistance of the winding calculated using [5]; and $R_{e}$ is the resistance due to the eddy current in the microwires that can be approximate using (1) as

$$
R_{e}=\omega \mu_{0} \mu_{\mu w}^{\prime} \frac{A_{\mu w}}{l_{\mu w}} \operatorname{Im}(F(\Delta)) .
$$

The real permeability and the magnetic loss tangent are represented in Fig. 10.

\section{B. Impedance Analyzer}

In this case, to characterize the microwires, the secondary winding used in the previous method is removed. In this way, 


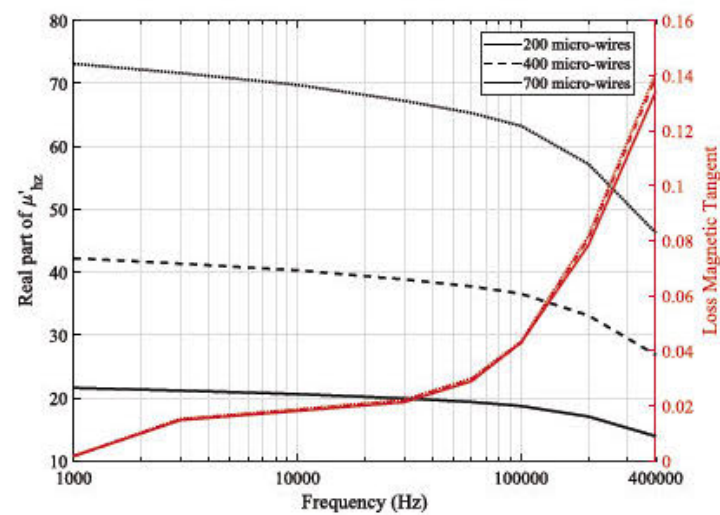

(a)

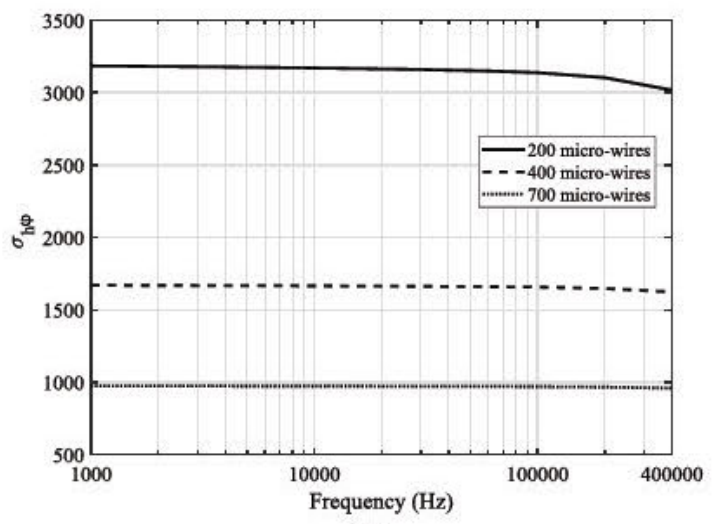

(b)

Fig. 11. Illustration of: (a) real part of the complex permeability of the homogeneous microwire needed to conduct the simulation by FE; and (b) conductivity of the homogeneous microwire needed to perform the FE analysis.

a simple coil has been used as can be seen in Fig. 9(c). The dimensions and the number of microwires are the same.

The impedance equation can be expressed as

$$
Z=R+j \omega L_{s} .
$$

Due to the permeability, which has two components: real (energy) and imaginary (losses), the expression (31) can be rewritten in terms of complex inductance (real and imaginary)

$$
Z=R+j \omega\left(L_{R}+L_{\mathrm{IM}}\right)
$$

where

$$
\begin{aligned}
L_{R} & =\frac{\mu_{r}^{\prime} \mu_{0} N_{1}^{2} A_{\mu w}}{l_{c}}+\frac{\mu_{0} N_{1}^{2} A_{\text {air }}}{l_{c}} \\
L_{\mathrm{IM}} & =\frac{-j \mu_{r}^{\prime \prime} \mu_{0} N_{1}^{2} A_{\mu w}}{l_{c}} .
\end{aligned}
$$

Thus, the impedance of the microwires may be given by

$$
Z=R+\frac{\omega \mu_{\mu w}^{\prime \prime} L_{R}}{\mu_{\mu w}^{\prime}}+j \omega L_{R}
$$

The real permeability of the microwires may be given by

$$
\mu_{\mu w}^{\prime}=\frac{l_{c} L_{R}}{\mu_{0} N_{1}^{2} A_{\mu w}}-\frac{A_{\text {air }}}{A_{\mu w}} .
$$

Thus, the imaginary permeability of the microwires may be given by

$$
\mu_{\mu w}^{\prime \prime}=\frac{l_{c}\left(R_{c}-R_{w}-R_{e}\right)}{\omega \mu_{0} N_{1}^{2} A_{\mu w}}
$$

where $R_{c}, R_{w}$, and $R_{e}$ are the resistance due to the core (total resistance), winding, and eddy current in the microwires, respectively, calculated as in the previous case.

The real permeability and the loss tangent are represented in Fig. 10.

\section{Measured Conductivity}

The conductivity of the microwires has been obtained by measuring the dc resistance of different samples of microwire based on the same composition. The approximate value obtained is $695000 \mathrm{~S} / \mathrm{m}$.

\section{FE Modeling Results}

Since the complex relative permeability and conductivity are known (see Fig. 10), various prototypes are measured and simulated in Ansys Maxwell using (10), (13), (14), (22), and (23).

The dimensions of the antennas are the same, but the number of microwires: $120 \mathrm{~mm} \times 9 \mathrm{~mm} \times 5 \mathrm{~mm}$. The different magnetic cores are made of 200,400 , and 700 microwires characterized in the previous section. The 71-turn winding is made of copper wire with a diameter of $0.45 \mathrm{~mm}$.

The finite element simulations have been performed assuming axisymmetric boundaries. A homogeneous microwire is defined, as shown in Fig. 13(a). Due to the inherent nature of the antenna, a significant part of the flux goes in the perpendicular direction. To perform these simulations, all parameters that the model provides are needed to model the homogeneous microwire: real and imaginary permeability in $z$-direction and $\rho$-direction; and conductivity in $\varphi$-direction.

Fig. 11 shows the homogeneous microwires' magnetic parameters in $z$-direction and electric parameter that the simulation needs to be conducted.

The winding's conductors are defined by 71 circles of conductivity $58 \mathrm{MS} / \mathrm{m}$. The net current through the conductors will be $12 \mathrm{~A}$ of peak. The diameter is set in $0.45 \mathrm{~mm}$. It can be seen in Fig. 13(a). Since these magnetic microwires will be used as magnetic cores in RFID antennas, the frequency varies from 1 to $400 \mathrm{kHz}$.

The measured inductance and ac resistance compared with the simulated results are shown in Fig. 12.

As shown in Fig. 12(a)-(c) (antennas made with 200, 400, and 700 microwires), the simulations show a good agreement to predict the ac resistance along the frequency range simulated, also when (2) is simplified for higher frequencies. In the inductance results cases, the FE simulations present a good agreement in frequency from 1 to $120 \mathrm{kHz}$. However, due to the fact that (2) is simplified and it is not valid for higher frequencies, the model underpredicts the inductance of the inductive magnetic coils with open magnetic-paths.

To the best of authors' knowledge, this is the first magnetic model to approximate the magnetic and electrical parameters of these kinds of magnetic material to allow fast macroscopic simulations in an FE tool. These simulations take few seconds and can predict, in a macroscopic sense, the behavior of these 


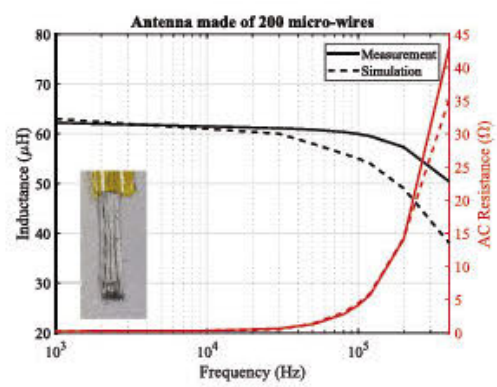

(a)

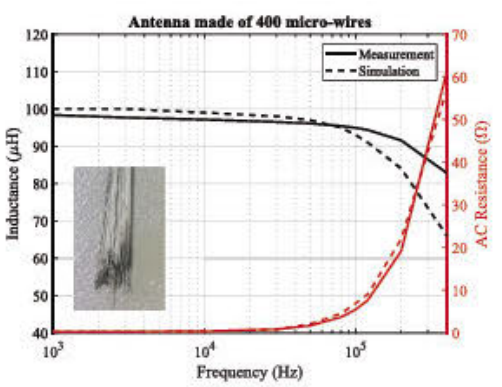

(b)

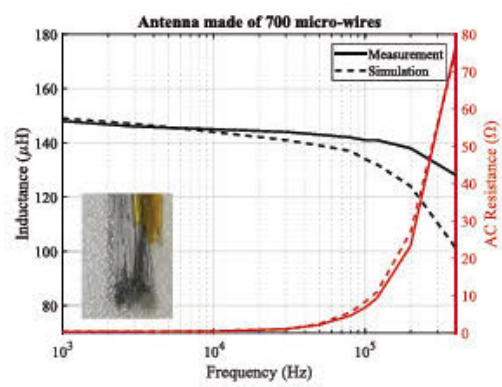

(c)

Fig. 12. Inductance and resistance of antenna with: (a) 200 microwires; (b) 400 microwires; and (c) 700 microwires.

TABLE I

COMPUTATIONAL TIME AND MESH REQUIRED

\begin{tabular}{|c||c|c|}
\hline $\mathbf{f}(\mathbf{k H z})$ & Simulation Time (sec) & Mesh (Triangles) \\
\hline \hline $\mathbf{1}$ & 24 & 6584 \\
\hline $\mathbf{1 0}$ & 25 & 6621 \\
\hline 100 & 25 & 6686 \\
\hline $\mathbf{4 0 0}$ & 27 & 7072 \\
\hline
\end{tabular}

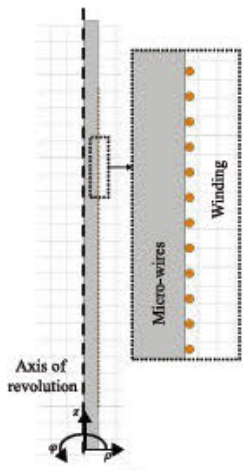

(a)

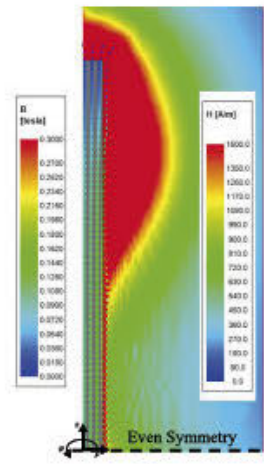

(b)
Fig. 13. Illustration of: (a) FE Simulation setup in Ansys Maxwell; (b) magnetic field distribution inside and outside of the antenna made of 400 microwires.

antennas. Table I reveals that the computational time is around $26 \mathrm{~s}$ per frequency, even if it is needed a larger mesh for both the magnetic microwire compound and the winding.

Regarding Fig. 13(b), where an RFID antenna made of 400 microwires at $50 \mathrm{kHz}$ excited by $12 \mathrm{~A}$ of peak is simulated, it can be noticed that the magnetic field $B$ inside of the magnetic core is mainly oriented in the $z$-direction. The reason for this fact is the high permeability that these microwires have in the $z$-direction and the low permeability in $\rho$-direction.

In order to compare this material with a common ferrite, an FE simulation at $20 \mathrm{kHz}$ is performed where two RFID antennas are simulated: 1) a magnetic core made of ferrite, and 2) a magnetic core made of 65000 microwires (that occupies the same area than the ferrite). The ac resistance is equal, but the inductance is higher in the microwires case ( $8 \%$ higher). Because of that, the radiated magnetic field is higher in the microwires case. It can be seen in Fig. 14.

\section{CONCLUSION}

In this article, a method to allow FE simulation of compound materials with magnetic microwires is proposed. The method is based on the use of a homogeneous material with anisotropic

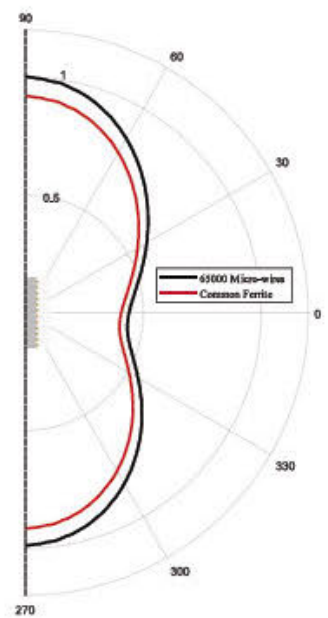

Fig. 14. Polar representation of the magnetic field radiated normalized of an RFID antenna made of microwires versus ferrite.

properties that keeps macroscopic energy and losses of the compound material in axial and radial directions. The physical properties of the homogeneous material are obtained by means of analytical equations. The model allows FE simulations of magnetic cores made of magnetic microwires; these simulations can be performed in a few seconds due to the simplicity of the model defined in the FE tool.

Several prototypes are constructed to test the models. The measurement of these prototypes and the FE simulations results are compared. The model presents good results to predict the ac resistance of the antennas made of microwires in the range analyzed. On the other hand, the inductance results do not present the same accuracy. The three samples measure present the same behavior. From 1 to $100 \mathrm{kHz}$, the maximum error in the energy is around $6 \%, 1 \%$, and $1 \%$ for 200,400 , and 700 microwires, respectively. From 100 to $400 \mathrm{kHz}$, the simulated inductance is underpredicted, and the average error is round $10 \%$. The model presents good results when used under the assumption that the skin depth of the microwires is higher than the diameter of the microwires, and the length of the microwires compared to the radius can be assumed to be infinite.

Overall, these results obtained by the model proposed are relevant to understanding the performance of these materials as a magnetic core in RFID antennas. Although the capability of these materials to handle with mechanical stresses is very high, they have high magnetic losses that make unsuitable this material (based on the alloy studied) for high frequencies. 


\section{REFERENCES}

[1] D. He and M. Shiwa, "A magnetic sensor with amorphous wire," Sensors, vol. 14, no. 6, pp. 10644-10649, 2014.

[2] T. K. Das, S. K. Mandal, A. K. Panda, S. Bhattacharya, P. Banerji, and A. Mitra, "Giant magnetoimpedance (GMI) effect and field sensitivity of ferrofluid coated co66fe2si13b15cr4 soft magnetic amorphous microwire," Phys. Procedia, vol. 54, pp. 16-22, 2014.

[3] G. I. Fernández, Propiedades Magnéticas Y De Transporte De Nuevos Microhilos Mono y Bifásicos, Ph.D. thesis, Departamento de Física de la Materia Condensada, Universidad Autónoma de Madrid, Málaga, Spain, 2010.

[4] J. Devkota et al., "A soft ferromagnetic multiwire-based inductance coil sensor for sensing applications," J. Appl. Phys., vol. 116, no. 23, 2014, Art. no. 234504.

[5] A. Delgado, G. Salinas, J. Rodríguez, J. A. Oliver, and J. A. Cobos, "Finite element modelling of litz wire conductors and compound magnetic materials based on magnetic nano-particles by means of equivalent homogeneous materials for wireless power transfer system," in Proc. IEEE 19th Workshop Control Model. Power Electron., 2018, pp. 1-5.

[6] J. Wu and Y. Lei, "Analytical expression to impedance for solenoid coil with a coaxial cylindrical ferrite core of finite length," J. Phys. D: Appl. Phys., vol. 35, no. 6, pp. 570-575, 2002.

[7] T. Fawzi, K. Ali, and P. Burke, "Eddy current losses in finite length conducting cylinders," IEEE Trans. Magn., vol. MAG-19, no. 5, pp. 2216-2218, Sep. 1983.

[8] H. Sabbagh, "A model of eddy-current probes with ferrite cores," IEEE Trans. Magn., vol. MAG-23, no. 3, pp. 1888-1904, May 1987.

[9] W. G. Hurley and W. H. Wölfle, Transformers and Inductors for Power Electronics: Theory, Design and Applications. Hoboken, NJ, USA: Wiley, 2013.

[10] A. Stadler, "Radiated magnetic field of a low-frequency ferrite rod antenna," in Proc. IEEE 7th Int. Conf.-Workshop Compat. Power Electron., 2011, pp. 283-288.

[11] R. Prieto, L. Ostergaard, J. A. Cobos, and J. Uceda, "Axisymmetric modeling of 3d magnetic components," in Proc. 14th Annu. Appl. Power Electron Conf. Expo. Conf. Proc., Mar. 1999, vol. 1, pp. 213-219.

[12] R. Prieto, J. A. Cobos, O. Garcia, P. Alou, and J. Uceda, "Study of 3d magnetic components by means of "double 2-d" methodology," IEEE Trans. Ind. Electron., vol. 50, no. 1, pp. 183-192, Feb. 2003.

[13] A. Delgado, J. A. Oliver, J. A. Cobos, and J. Rodríguez, "Axisymmetric finite element modelling of compound magnetic materials based on magnetic micro-wires by means of equivalent homogeneous material," in Proc. IEEE 18th Workshop Control Model. Power Electron., 2017, pp. $1-5$.

[14] J. Muhlethaler, J. W. Kolar, and A. Ecklebe, "A novel approach for 3d air gap reluctance calculations," in Proc. IEEE 8th Int. Conf. Power Electron. Asia, 2011, pp. 446-452.

[15] A. Balakrishnan, W. T. Joines, and T. G. Wilson, "Air-gap reluctance and inductance calculations for magnetic circuits using a Schwarz-Christoffel transformation," IEEE Trans. Power Electron., vol. 12, no. 4, pp. 654-663, Jul. 1997.

[16] Z. Hashin and S. Shtrikman, "A variational approach to the theory of the effective magnetic permeability of multiphase materials," J. Appl. Phys., vol. 33, no. 10, pp. 3125-3131, 1962 .

[17] Z. Szabó, G.-H. Park, R. Hedge, and E.-P. Li, "A unique extraction of metamaterial parameters based on Kramers-Kronig relationship," IEEE Trans. Microw. Theory Tech., vol. 58, no. 10, pp. 2646-2653, Oct. 2010.

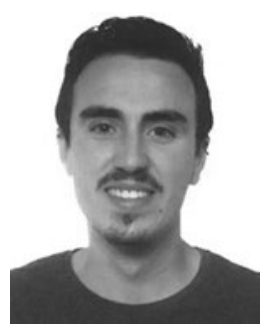

Alberto Delgado (Student Member, IEEE) received the B.Sc. degree in electrical engineering in 2016 from the University of Málaga (UMA), Málaga, Spain, and the M.Sc. degree in industrial electronics in 2017 from the Universidad Politécnica de Madrid (UPM), Madrid, Spain, where he is currently working toward the $\mathrm{Ph} . \mathrm{D}$. degree in industrial electronics.

He became Teaching Assistant in 2019 at the Universidad Politécnica de Madrid, Madrid, Spain. His research activities include modeling of $\mathrm{dc}-\mathrm{dc}$ converters for the inductive power transfer system, magnetic components for different applications such as RFID communications and wireless charging, and magnetic nanomaterials and micromaterials.

Dr. Delgado was awarded honors on several occasions and he achieved the Best Student of the Year Award, during his undergraduate studies.

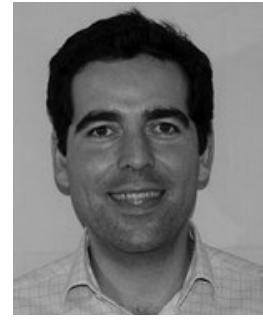

Jesús Ángel Oliver (Member, IEEE) received the doctoral and master's degrees in electrical engineering from the Universidad Politécnica de Madrid (UPM), Madrid, Spain, in 1996 and 2007, respectively.

He was an Assistant Professor in 2001 and an Associate Professor with the UPM in 2007. He has been author and co-author in more than 150 scientific papers on Journals and Conferences and he holds five patents. His research activities include modeling (dc-dc converters, magnetic components, piezoelectric transformers, fuel-cells, and de distributed power electronic systems), fast control techniques for dc-dc converters for VRM applications and RF amplifiers, three-phase rectifiers for aircraft applications, wireless power transfer, and power systems on Chip.

Dr. Oliver has led numerous research projects with private and public funding and he has participated in more than 50 direct R\&D projects with companies in Europe, US, Australia, and China. He is currently an Associate Editor for the IEEE TRANSACTIONS ON POWER EleCtronics.

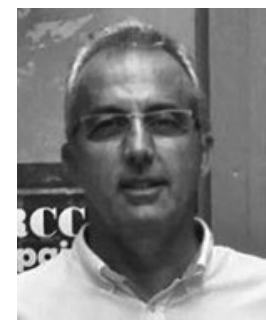

José Antonio Cobos (Fellow, IEEE) is currently a Full Professor with the Universidad Politécnica de Madrid (UPM), Madrid, Spain. In 2006, he was the Founder Director of the Centro de Electrnica Industrial, UPM (CEI-UPM), a university research center leading a strong industrial program in power electronics and digital systems. Since 2016, he has been the Founder President in Industrial Council of UPM, to coordinate education and research with industry. His contributions are focused on power supply systems for industrial, aerospace, telecom, automotive, renewable energy, and medical applications. He advised more than 50 graduate students, has authored or coauthored more than 300 technical papers ( $>8000$ citations, $h=47$ ), and is co-inventor of patents with six companies. His current research interests include energy efficiency in digital systems, RF amplifiers, renewable energy, magnetic components, transcutaneous energy transfer, and biomedical applications.

Dr. Cobos was an Adcom Member of the Power Electronics Society (IEEEPELS) and the Chair of its Technical Committee on DC Power Supply systems. He was the General Chair of PwrSoC 2016 (IEEE-PELS and Power Supply Manufacturers Association, PSMA) and an Associate Editor for the IEEE TRANSACTIONS ON POWER ELECTRONICS and the PELS Letters. He conducted several professional seminars and tutorials in USA, U.K., Austria, Germany, Italy, Sweden, Switzerland, Syria, Mexico, and Macedonia. He is a Steering Committee Member (Program Chair in 2019) of the IEEE Applied Power Electronics Conference (APEC). In 2016-2017, he was an RCC Fellow a Harvard University, Cambridge, MA, USA, and a Fulbrighter with the University of California at Berkeley, Berkeley, CA, USA.

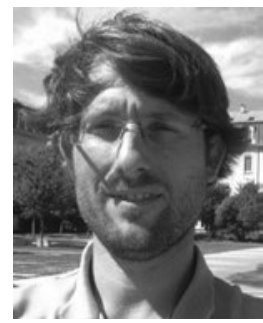

Jorge Rodriguez-Moreno was born in Málaga, Spain, in 1984. He received the B.S. and M.S. degrees in industrial engineering from Malaga University, Málaga, Spain, 2009 and 2010, respectively, and the Ph.D. degree in applied physics from Universidad de Málaga, in 2014

Since 2015, has been with the Innovation Center, Premo Group, Málaga, Spain, as an Engineer. During his Ph.D. degree, he was a Visiting Researcher with the Physics Institute of Freiburg, Freiburg, Germany, and with the Lawrence Berkeley National Laboratory, Berkeley, CA, USA, during his Postdoctoral. His research interests include magnetic sensor, RFID components, materials characterization, and supercapacitors.

Dr. Rodriguez-Moreno received the SUSChem Award as a co-author for one of his publications. 